\title{
Comprehensive Analyses of White-Handed Gibbon Chromosomes Enables Access to 92 Evolutionary Conserved Breakpoints Compared to the Human Genome
}

\author{
Anja Weise $^{\mathrm{a}}$ Nadezda Kosyakova ${ }^{\mathrm{a}}$ Martin Voigt ${ }^{\mathrm{a}}$ Nadine Aust $^{\mathrm{a}}$ \\ Kristin Mrasek $^{a}$ Sharon Löhmer ${ }^{a}$ Nikolai Rubtsov $^{b}$ Tatyana V. Karamysheva ${ }^{b}$ \\ Vladimir A. Trifonov c, d David Hardekopfe Tereza Jančuškováe Sona Pekova ${ }^{e}$ \\ Kathleen Wilhelm ${ }^{\mathrm{a}}$ Thomas Liehr ${ }^{\mathrm{a}}$ Xiaobo Fan ${ }^{\mathrm{a}}$ \\ anstitute of Human Genetics, Jena University Hospital, Friedrich Schiller University, Jena, Germany; \\ ${ }^{b}$ Institute of Cytology and Genetics, ${ }^{C}$ Institute of Molecular and Cellular Biology SB RAS, and

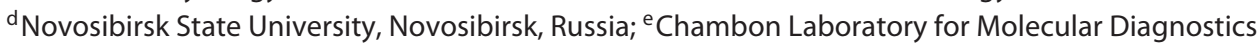 \\ (member of the Synlab Czech Laboratory Group), Prague, Czech Republic
}

\section{Key Words}

Comparative genomics · Evolutionary conserved

breakpoints $\cdot$ Hylobates lar . Molecular cytogenetics

\begin{abstract}
Gibbon species (Hylobatidae) impress with an unusually high number of numerical and structural chromosomal changes within the family itself as well as compared to other Hominoidea including humans. In former studies applying molecular cytogenetic methods, 86 evolutionary conserved breakpoints (ECBs) were reported in the white-handed gibbon (Hylobates lar, HLA) with respect to the human genome. To analyze those ECBs in more detail and also to achieve a better understanding of the fast karyotype evolution in $\mathrm{Hy}$ lobatidae, molecular data for these regions are indispensably necessary. In the present study, we obtained whole chromosome-specific probes by microdissection of all 21 HLA autosomes and prepared them for aCGH. Locus-specific DNA probes were also used for further molecular cytogenetic characterization of selected regions. Thus, we could map
\end{abstract}

6 yet unreported ECBs in HLA with respect to the human genome. Additionally, in 26 of the 86 previously reported ECBs, the present approach enabled a more precise breakpoint mapping. Interestingly, a preferred localization of ECBs within segmental duplications, copy number variant regions, and fragile sites was observed.

(c) 2015 S. Karger AG, Basel

The superfamily Hominoidea consists of great (orangutans, gorillas, chimpanzees, and humans) and lesser (gibbons) apes. The evolutionary paths of human (Homo sapiens sapiens, HSA) and gibbon are closely linked by a common ancestor living between 15 and 20 million years ago [Müller et al., 2003; Perelman et al., 2011]. In contrast to the family of great apes, gibbons demonstrate a high number of chromosomal rearrangements including inversions, translocations, fissions, and fusions [Brown and O’Neill, 2009]. Overall, gibbon species differ greatly in their chromosomal constitution, even between the 4 extant genera: Hoolock $(2 \mathrm{n}=38)$, Hylobates $(2 \mathrm{n}=44)$, Sym-

\section{KARGER 125}

(c) 2015 S. Karger AG, Basel

$1424-8581 / 15 / 1451-0042 \$ 39.50 / 0$

E-Mail karger@karger.com

www.karger.com/cgr
Anja Weise

Institute of Human Genetics

Jena University Hospital, Friedrich Schiller University

Postfach, D-07740 Jena (Germany)

E-Mail Anja.Weise@med.uni-jena.de 
phalangus $(2 \mathrm{n}=50)$, and Nomascus $(2 \mathrm{n}=52)$ [Müller et al., 2003]. There are also profound differences among the Hylobates group. For example, since the separation of $H$. lar and $H$. syndactylus, these 2 subspecies have independently accumulated 14 and 16 translocations within their group, respectively [O'Brien et al., 2006].

Despite the close relation to humans and the great apes, the classical banding pattern of gibbon chromosomes showed only few homologies between great and lesser apes, except for the X chromosome which is well conserved. On the other hand, the karyotypes of Old World monkeys, such as macaques and baboons, are very close to the ancestral primate karyotype and thus share most of their karyotype structure with the great apes [Stanyon et al., 2008].

The white-handed gibbon (H. lar, HLA) has 44 chromosomes, remarkably without a single acrocentric pair [Mrasek et al., 2003]. However, compared to human and most other primates, HLA shows an exceptionally high rate of chromosomal rearrangements. Several studies were previously done to investigate the chromosomal organization of HLA. In 1975, Tantravahi and colleagues carried out a cytogenetic study based on quinacrine, trypsin-Giemsa, and centromeric heterochromatin stains. Yet, they could only show how different HLA was in comparison to human and some other great apes. Jauch et al. [1992] used chromosome painting to detect the homologies between gibbon, great apes, and human and demonstrated a high degree of karyotype reshuffling in gibbon. Müller et al. [2003] applied human chromosome-specific probes to study homologous regions in all 4 hylobatid genera and to reconstruct the chromosomal evolution within the family. Multicolor banding was applied by Mrasek et al. [2003], revealing 71 breakpoints present in HLA compared to human. Recently, Misceo et al. [2008] performed a vast study using over 1,000 human BAC clones to determine the extension, reciprocal arrangement, and orientation of chromosomal segments of HLA compared to HSA. They ended up with a total number of 86 evolutionary conserved breakpoints (ECBs). More molecular data on ECBs were published recently by Carbone et al. [2014] from total sequencing of $H$. pileatus and $H$. moloch.

Since the chromosome rearrangement rate of gibbons is at least an order higher in magnitude compared to the average rearrangement rate in mammals [Zhao et al., 2004; Misceo et al., 2008], investigating the HLA genome could provide a better understanding of the mechanisms of genome plasticity in terms of species evolution. In fact, ECBs in HLA might correspond to human regions encompassing large amounts of duplicated genes [Carbone et al.,
2009]. Moreover, physical clustering of gene families may be explained, or further insights might be obtained why some genes remain conserved over millions of years while others evolved more quickly [O'Brien and Yuhki, 1999]. So far, no excessive analysis of HLA samples using aCGH has been accomplished. The higher resolution of this method should generate a more complete picture than cytogenetics or molecular cytogenetics alone and is also able to detect submicroscopic, previously hidden, rearrangements [Gribble et al., 2009; Capozzi et al., 2012; Liehr et al., 2013].

\section{Methods}

\section{Material}

A female HLA cell line, provided by Prof. M. Rocchi (Bari, Italy) [Mrasek et al., 2003], was used in this study. As far as applicable, we state that (a) the research complied with protocols approved by the appropriate Institutional Animal Care Committee of the University of Jena, who declared that they are not responsible for cell line studies; (b) the research adhered to the legal requirements of Germany; and (c) the research adhered to the American Society of Primatologists (ASP) Principles for the Ethical Treatment of NonHuman Primates.

\section{Microdissection}

Chromosomes of the HLA cell line were subjected to glass-needle-based microdissection as described previously [Yang et al., 2009]. A total of 10-15 copies of each HLA autosome were microdissected, DOP-PCR amplified, labeled, and used for reverse FISH to prove the specificity and quality before aCGH was applied. Apart from HLA chromosomes 1 and 7, FISH-based microdissection was used for HLA probe preparation as previously reported [Kosyakova et al., 2013].

\section{$a C G H$}

DOP-PCR-amplified and labeled HLA-derived DNA was applied in aCGH as previously reported for human partial chromosome paints [Weise et al., 2008]. aCGH analysis was performed using $4 \times 180 \mathrm{~K}$ SurePrint G3 Human CGH microarray slides (Agilent Technologies, Santa Clara, Calif., USA). These slides were produced via the Agilent 60-mer SurePrint technology containing 170,334 60-mer oligonucleotide probes that cover the entire human genome with a $13-\mathrm{kb}$ overall median probe spacing. Probes were processed following Agilent's 'Oligonucleotide Array-Based CGH for Genomic DNA Analysis' protocol (v.6.2). Two fluorescence dyes were used to label references and samples. The arrays were scanned after hybridization using a dual-color laser scanner. Images were subsequently processed by Agilent's Feature Extraction v10.5 and analyzed using Agilent Workbench 5.0 (NCBI36/hg18).

\section{Molecular Cytogenetics}

FISH was performed using commercially available probes according to the manufacturer's instructions. The following specific subtelomeric probes were applied: 1pter, 4pter, 4qter, 5qter, 6qter, 7qter, 8pter, 8qter, 9pter, 10pter, 10qter, 12pter, 12qter, 14qter, $15 q$ ter, 16 pter, 16qter, 17qter, 19pter,20pter, 20qter, Xpter, Xqter (Kreatech, Amsterdam, The Netherlands); and 1qter, 2pter, 2qter, 
Fig. 1. A Schematic drawing of HLA array results with respect to the human karyotype. B Schematic HLA karyogram showing the orientation of the chromosomal blocks being syntenic to HSA. The scheme is drawn according to data presented in this work together with data from Marsek et al. [2003] and Misceo et al. [2008].

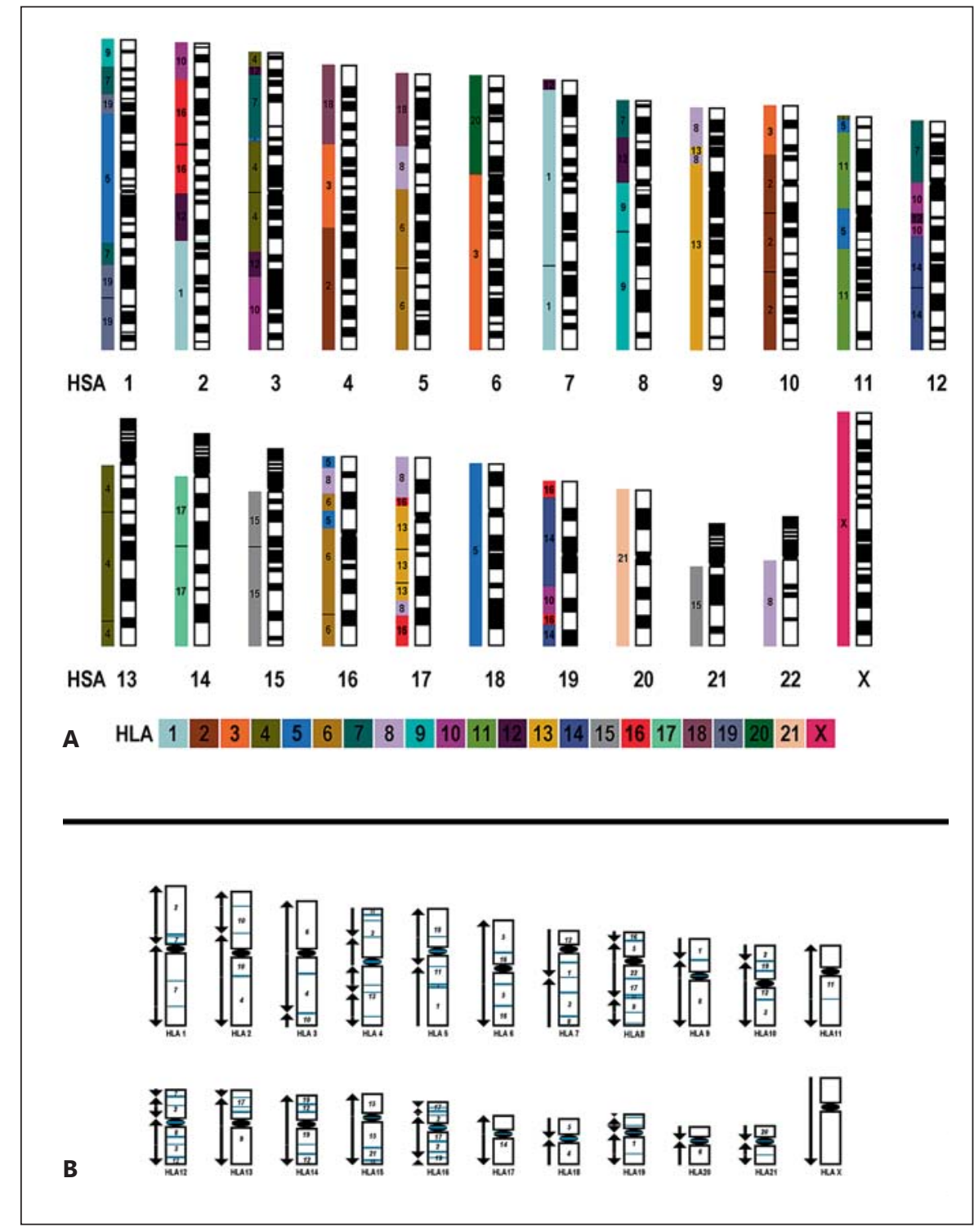

3 pter, 3qter, 5pter, 6pter, 7pter, 9pter, 9qter, 11pter, 11qter, 13qter, 15qter, 17pter, 18pter, 18qter, 19qter, 21qter, 22qter (Abbott/Vysis, Wiesbaden, Germany). BACs from BACPAC Chori were used for further characterization of the following regions: $1 \mathrm{p} 36,1 \mathrm{p} 36.21$, $3 q 29,9 p 21,9 q 34.3,11 q 13,16 \mathrm{p} 11.2,16 \mathrm{p} 13,17 \mathrm{p} 11.2,17 \mathrm{q} 23$, and 20q13. FISH was done according to standard procedures [Mrasek et al., 2003]. The evaluation was done using a Zeiss Axioplan fluorescence microscope (Zeiss, Jena, Germany) with MetaSystems (Isis) software (Altlussheim, Germany). A total of 10-20 metaphases per probe were analyzed.

\section{Databases}

All human genome mapping calculations, assignments to cytogenetic bands, and sequence analyses were done according to the UCSC database version March 2006 (NCBI36/hg18, http://genome.ucsc.edu/cgi-bin/hgGateway). Each ECB determined by
aCGH was analyzed for copy number variant regions (CNVs) and/ or segmental duplications (SDs) and known disease causing regions. As the mean resolution of the used aCGH approach was 26 $\mathrm{kb}, \pm 25 \mathrm{~kb}$ was the minimum size of the region analyzed in the databases for each of the ECBs analyzed.

\section{Results and Discussion}

In the present study, for the first time the combined microdissection/aCGH approach [Backx et al., 2007; Weise et al., 2008; Villa et al., 2011; Jancuskova et al., 2013] was successfully applied to narrow down 43 euchromatic autosomal ECBs in HLA (fig. 1). Additionally,
44

Cytogenet Genome Res 2015;145:42-49 DOI: $10.1159 / 000381764$
Weise et al. 


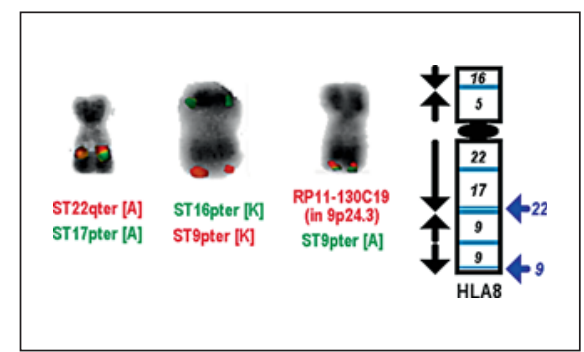

Fig. 2. Application of human subtelomeric probes identified 3 new rearrangements not reported before (FISH pictures and blue arrows) for HLA chromosome 8. Black arrows along the karyogram represent the orientation of the corresponding chromosome fragments with homologous HSA fragments. [A] = Vysis $/$ Abbott probe; $[\mathrm{K}]=$ Kreatech probe; $\mathrm{ST}=$ subtelomeric probe.

human subtelomeric probes were applied to confirm and/ or refine the aCGH data (fig. 2; online suppl. tables 1, 2; see www.karger.com/doi/10.1159/000381764 for all online suppl. material). The data obtained here (original data of aCGH is exemplarily depicted in figure 3 ) were evaluated together with previously published own data [Mrasek et al., 2003] and with results of other authors [Misceo et al., 2008]. Thus, in online suppl. tables 3 and 4 , the best presently available approximation to $92 \mathrm{ECBs}$ in HLA is presented. In figure 1, these data are summarized comparing syntenic blocks between HSA and HLA. Even though meanwhile newer versions than NCBI36/ hg18 are available, this version had to be used for this work as numerous BAC probes are no longer listed in current versions like GRCh37/hg19 or GRCh38/hg38.

\section{Novel Submicroscopic Chromosomal Changes and}

Previously Undetected Breakpoints

As aCGH is not suited to detect balanced translocations in a whole genomic setting, we combined glass-needle-based microdissection with aCGH to refine ECB mapping in HLA with respect to the human karyotype. The combined microdissection/aCGH approach was applied previously for the exact sizing of partial chromosome paints [Weise et al., 2008] or the precise determination of chromosomal breakpoints [Backx et al., 2007; Villa et al., 2011; Jancuskova et al., 2013]. HLA chromosomes of interest were painted by specifically labeled human DNA probes, and FISH microdissection of the labeled chromosomes was done. The obtained DNA libraries were amplified by DOP-PCR and applied in aCGH. To verify suspect and weakly covered aCGH regions like subtelomeric regions, we applied all available 41 human sub-

Comprehensive Karyotype of

White-Handed Gibbon telomeric probes and additional locus-specific human BAC probes in 2- to 3-color-FISH settings to HLA chromosomes derived from a female HLA cell line.

As shown in online suppl. tables 3 and 5, this combined approach was able to detect the following yet unreported breakpoints: 3-4, 9-5 (fig. 2), 16-1, 16-3, 16-4, and 22-4 (figs. 2, 4). On the other hand, BAC-FISH, being able to resolve ECBs involved in balanced as well as in unbalanced rearrangements as applied by Misceo et al. [2008], could identify the following 37 breakpoints not detectable by aCGH: $1-3,1-4,1-8,2-2,2-3,3-6,5-3$, $6-1,6-3,7-2,7-3,7-4,7-5,7-6,8-3,10-2,10-3,12-5$, $13-2,13-3,13-4,14-2,15-2,15-3,16-5,17-4,17-5,17-$ $8,17-9,18-1,18-2,19-5,20-1,20-2,20-3,22-3$, and $22-4$.

For breakpoints 3-1, 9-3, 9-4, and 11-1 another problem of aCGH has to be considered. Those ECBs where not resolved in aCGH due to a low coverage of the corresponding regions in the assay itself. This shows that different approaches need to be performed and combined to obtain most comprehensive information on ECBs. Even in the age of next-generation-sequencing, balanced and complex rearrangements as well as repetitive heterochromatic regions and CNVs cannot unambiguously be resolved by an exclusive use of these modern approaches. Therefore, comparative chromosome painting of HSA and HLA provides a necessary overview on the extensive diversity within this group, such as chromosome numbers, composition of syntenic blocks, and type of translocations [Capozzi et al., 2012]. CNVs and fast mapping of euchromatic breakpoints is accomplished by the here applied approach of array painting. As a further step, nextgeneration whole-genome sequencing may provide additional insights into specific genetic information and functional mechanisms linked with large-scale changes during speciation [Carbone et al., 2014].

\section{Evolutionary Conserved Breakpoints and Copy \\ Number Variant Regions}

Detailed breakpoint analyses of the results summarized in online suppl. table 4 revealed that the majority of ECBs $(77 / 92 ; 83.7 \%)$ has already been reported to be involved in deleterious but viable deletions or duplications (which in most cases are larger than the analyzed breakpoint region) in human genetic diseases. Additionally, $~ 98.9 \%$ of the breakpoints are localized in CNVs, while only $50 \%$ of them are colocalized with SDs. This finding is in concordance with Carbone et al. [2006] who analyzed 100 ECBs in gibbons with a resolution of $200 \mathrm{~kb}$ and found that $46 \%$ of these breakpoints correspond to SDs with respect to the 
Fig. 3. Combined aCGH results for homologues regions of microdissected HLA chromosomes $7,10,12$, and 14 on human chromosome 12 (180k Agilent array).

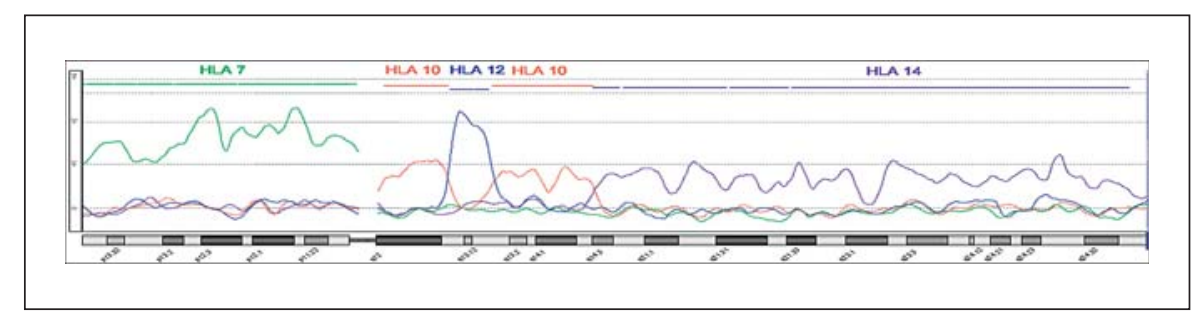

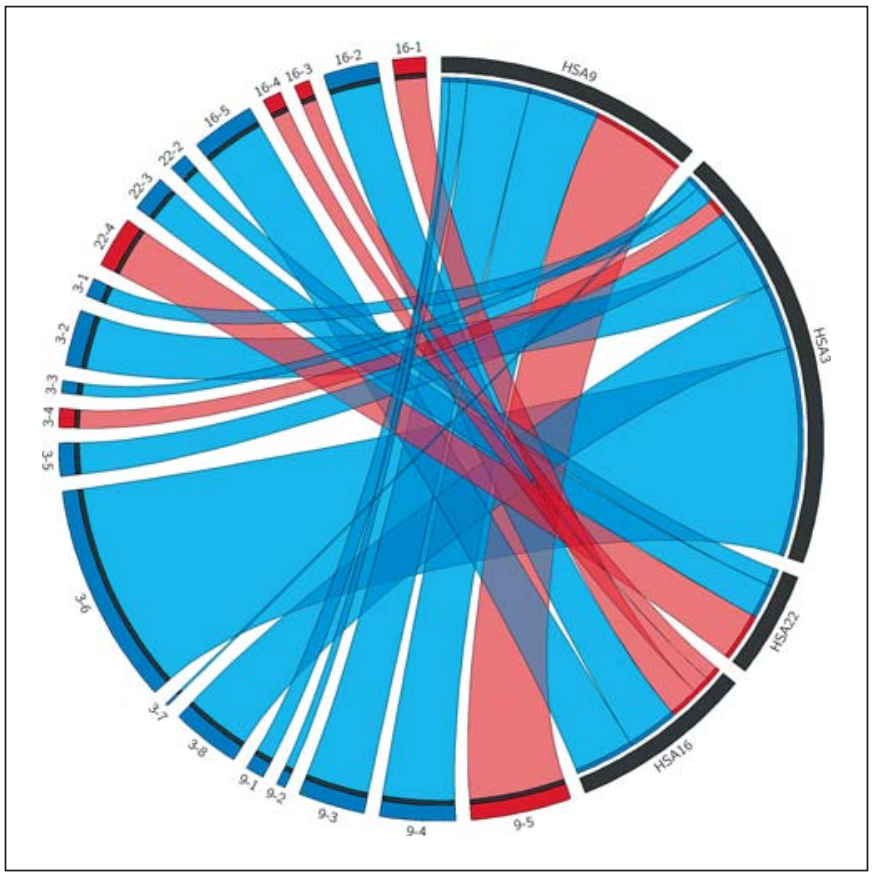

Fig. 4. Human chromosomes 3, 9, 16, and 22 with new identified ECBs in HLA are visualized by a circular diagram (created by http://mkweb.bcgsc.ca/circos/). Previously unreported breakpoints: $3-4,9-5$ (see also figure 2 ), 16-1, 16-3, 16-4, and 22-4 (fig. 2; online suppl. tables 3,4 ) are depicted in red, known ones in blue. The size of the region to which the ECBs were narrowed down are depicted as width of the interlink between HLA and HSA.

human sequence [Carbone et al., 2009; Capozzi et al., 2012]. Recent molecular data by Wilson et al. [2015] further underline the correlation and colocalization of CNVs, common fragile sites (CFSs), as well as ECBs. CFSs represent a special kind of genomic instability under replication stress. It is suggested that the latter may induce CNV formation. Thus, CNVs and CFSs may also be seen as different manifestations of perturbed replication dynamics in the corresponding loci. The observed SDs in ECBs could also be explained as a result of break-induced replication repair leading to enrichment of genomic duplications in these break-prone regions [Costantino et al., 2014]. Another interesting feature that underlines the reuse of ECBs as break-prone regions is their involvement in sequence rearrangements, like inversions, insertions, and sequence/ homology gaps being identified in other primates compared to the human genome (here analyzed for chimpanzee, orangutan, gorilla, rhesus monkey, and marmoset (online suppl. table 4)). Overall, ECBs may be interpreted as evolutionary highly active maybe due to a complex (epi)genetic architecture of those regions. The latter again stresses the necessity to combine various approaches for ECB mapping and studying genomic and karyotype evolution in as many species as possible.

\section{Evolutionary Conserved Breakpoints and OMIM \\ Genes}

In all analyzed ECBs, the gene density in only $3 / 92$ cases $(3.3 \%)$ is larger than 0.5 genes per $10 \mathrm{~kb}$, indicating that ECBs in HLA appear in more or less gene poor regions. When analyzing the closer surroundings of all identified autosomal ECBs (minimum $\pm 25 \mathrm{~kb}$ around each ECB, detailed results are shown in online suppl. tables 4 and 6), then $59 \%$ of the analyzed regions included overall 157 known OMIM genes. Furthermore, only 47 of those genes in ECB regions are known as disease causing.

In general, the influence of ECBs on the phenotype could be direct, i.e. due to interruption of genes, or indirect, due to position effects influencing the expression/ regulation of corresponding genes. Therefore, we analyzed the 154 identified OMIM genes in more detail for their function (online suppl. table 6) and found that $25 \%$ of them are highly expressed in the brain or are involved in neuronal function, $19 \%$ are somehow connected with cancer, $17 \%$ play a role for the function of the immune system, $10 \%$ are linked with intracellular trafficking, $8 \%$ each regulate/interact with DNA or are involved in metabolic processes, $5 \%$ influence the gestalt, $3 \%$ each are part of mitochondrial networks or are highly expressed in testis, and $2 \%$ are involved in cell cycle traits. The high degree
Weise et al. 
Fig. 5. ECBs and FSs are interlinked by a circular diagram (see also figure 4). Cytogenetically mapped FSs are depicted in yellow, molecular mapped FSs in red. The size of the region to which the FSs were narrowed down is depicted as width of the interlink between ECBs and FSs.

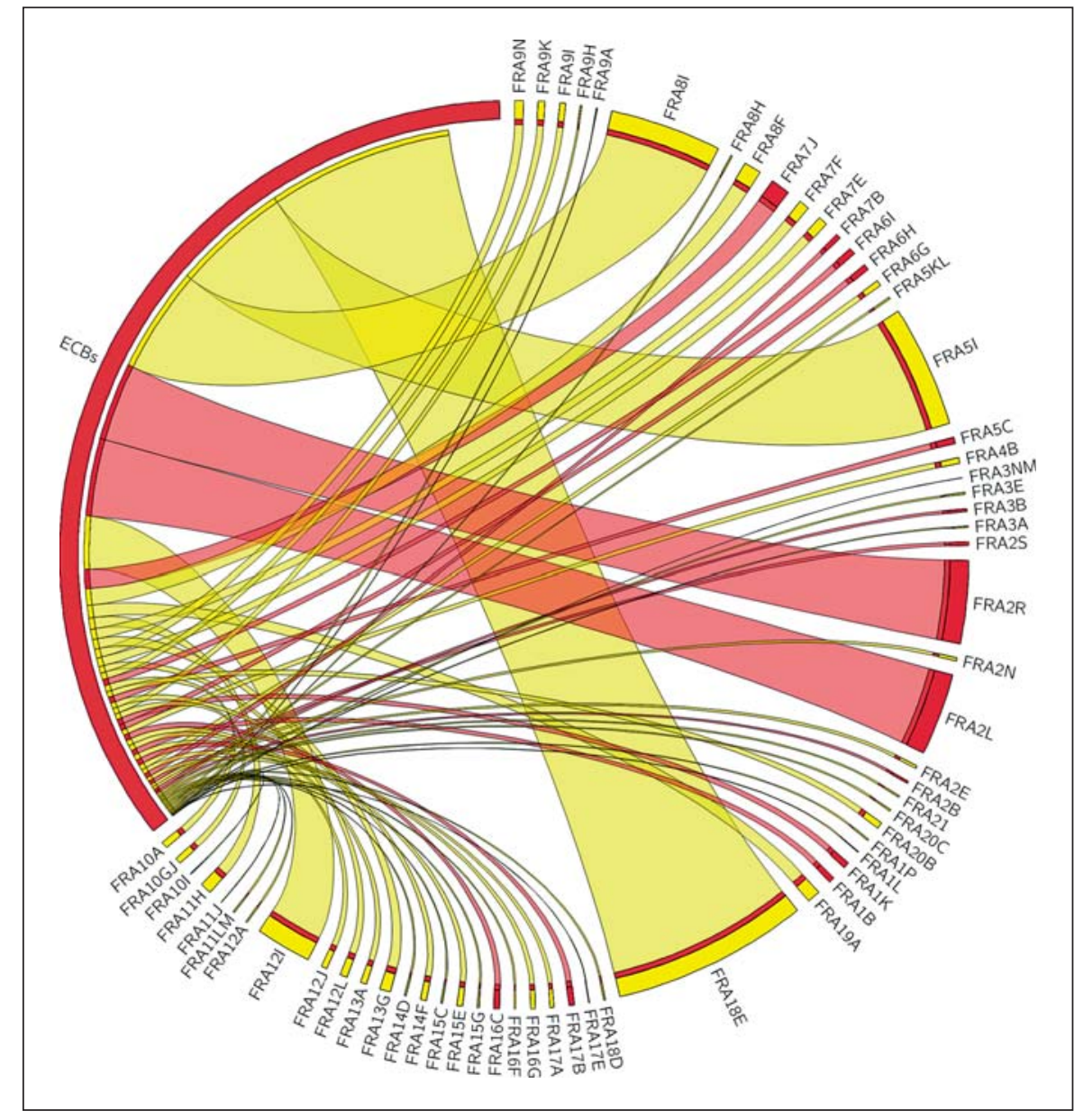

of association with brain-related genes and genes involved in the immune system are well documented and were previously discussed in the literature as a target for selection [Kehrer-Sawatzki and Cooper, 2007]. The identified OMIM genes which might have an influence concerning the differences in the gestalt are associated with growth (OMIM: 614145), obesity (OMIM: 606803), have skeletal (OMIM: 604032) and muscular functions (OMIM: 6009000), and affect hair keratin (OMIM: 602153) and skin physiology (OMIM: 607397 and 612121).

Concerning the lineage-specific high rate of chromosomal rearrangements in HLA, a well-controlled border between deleterious effects of chromosomal rearrangements (like in cancer) and well-controlled speciation must have taken place. Therefore, genes involved in cell cycle, DNA repair, and cancer development may be potential targets for this lineage-specific chromosome instability.

Interestingly, some of these genes play multiple roles like RBBP8 (RetinoBlastoma-Binding Protein 8, OMIM:
604124) which is involved in DNA regulation, cell cycle, DNA repair, and, therefore, also in cancer development. RBBP8 is found among several proteins that bind directly to the retinoblastoma protein which regulates cell proliferation. It is also associated with BRCA1 and is thought to modulate its functions in transcriptional regulation, DNA repair, and/or cell cycle checkpoint control. It is suggested that $R B B P 8$ may itself be a tumor suppressor acting in the same pathway as BRCA1. Mutations of $R B B P 8$ are found in patients with Seckel syndrome 2, Jawad syndrome, and patients with pancreatic carcinoma.

\section{Evolutionary Conserved Breakpoints and Common Fragile Sites}

CFSs can be induced in every individual and represent an intrinsic feature of human chromosome biology [Mrasek et al., 2010]. Furthermore, they are reported for several species like Drosophila [von Grotthuss et al., 
2010], mammals [Alekseyev and Pevzner, 2010], yeast [Rosen et al., 2013], or birds [Gordon et al., 2007]. We compared 230 common known human CFSs and rare FSs [Mrasek et al., 2010] together with 47 recently molecularly defined fragile site (online suppl. table 7) to underscore their role in evolutionary breakpoint reuse as suggested in a 'fragile breakage model of chromosome evolution' [Peng et al., 2006]. In the present as well as in other studies [Fan et al., 2013], a high rate of coincidence between ECBs and FSs [Mrasek et al., 2010] was observed: 65/92 (70.6\%) ECBs between HLA and HSA could be correlated with cytogenetic localization of FSs. For 12 of these 65 FSs, molecular data of their localization was available (online suppl. table 7), and all of them were in line with the suggestion of a colocalization of ECBs and FS (fig. 5). These data further support the hypothesis that chromosome rearrangements have not occurred randomly over the course of evolution but are focused preferentially within fragile regions.

\section{Genomic Sequence and Karyotype Evolution}

While ECBs share some common features (FSs, CNVs, $\mathrm{SDs}$ ), the comparison of available molecular breakpoint data in different gibbon species shows a high diversity, suggesting a more or less independent origin of ECBs within the gibbon family [Carbone et al., 2006, 2009, 2014; Roberto et al., 2007; Girirajan et al., 2009]. Still, this is investigated in more detail currently by high-resolution molecular and cytogenetic studies [Carbone et al., 2014]. Yet, in Hylobatidae, 10-20 fold faster chromosome rearrangement rates compared to most mammals [Misceo et al., 2008] were revealed, and similar rates were detected in muroid rodents [Romanenko et al., 2012] and equids [Trifonov et al., 2012]. The possible mechanisms responsible for such an increased genome evolution might be due to LAVA (gibbon specific retrotransposon)-induced premature transcription termination of chromosome segregation genes [Carbone et al., 2014]. Pericentric inversions are considered to be the most common euchromatic chromosomal differences between humans and the great apes [Nickerson and Nelson, 1998; Locke et al., 2003, Kehrer-Sawatzki et al., 2005]. According to fig. 1B, para- and pericentric inversions may be considered for HLA evolution for all but chromosome 18 and the $\mathrm{X}$ chromosome. Besides the recent thoughts published by Reinhard Stindl [2014] on altered chromosome fusion, inversion and separation during evolution might also be considered to explain specification events.

\section{Conclusion}

Overall, the currently available data did not change the suggestion that all hylobatid-specific rearrangements have a derived state and that these genomic changes have occurred after a common gibbon-ancestor split from the last common hominoid ancestor between 15 and 20 million years ago [Goodman, 1999; Perelman et al., 2011]. In more detail, half of the rearrangements that distinguish the hylobatid karyotypes from the human karyotype are shared by all 4 small ape genera and therefore occurred in their common ancestor. However, the breakpoint regions were narrowed down more precisely, which is necessary for further comparative studies in evolution research in Hominoidea and within gibbon subspecies.

\section{Acknowledgments}

This work was supported in part by DLR RUS 09/008 and the China Scholarship Council.

\section{References}

\footnotetext{
Alekseyer MA, Pevzner PA: Comparative ge- Capozzi O, Carbone L, Stanyon RR, Marra A, Carbone L, Harris RA, Gnerre S, Veeramah KR, nomics reveals birth and death of fragile re- Yang F, et al: A comprehensive molecular cygions in mammalian evolution. Genome Biol togenetic analysis of chromosome rearrange11:R117 (2010).

Backx L, Van Esch H, Melotte C, Kosyakova N, Starke H, et al: Array painting using microdis- Carbone L, Vessere GM, ten Hallers BF, Zhu B, sected chromosomes to map chromosomal Osoegawa K, et al: A high-resolution map of breakpoints. Cytogenet Genome Res 116: synteny disruptions in gibbon and human ge158-166 (2007).

Brown JD, O’Neill RJ: The mysteries of chromo- Carbone L, Harris RA, Vessere GM, Mootnick some evolution in gibbons: methylation is a AR, Humphray S, et al: Evolutionary breakprime suspect. PLoS Genet 5:e1000501(2009). points in the gibbon suggest association between cytosine methylation and karyotype evolution. PLoS Genet 5:e1000538 (2009).

Carbone L, Harris RA, Gnerre S, Veeramah KR,
Lorente-Galdos B, et al: Gibbon genome and the fast karyotype evolution of small apes. $\mathrm{Na}$ ture 513:195-201 (2014).

- Costantino L, Sotiriou SK, Rantala JK, Magin S, Mladenov E, et al: Break-induced replication repair of damaged forks induces genomic duplications in human cells. Science 343:88-91 (2014).

-Fan X, Pinthong K, Mkrtchyan H, Siripiyasing P, Kosyakova N, et al: First detailed reconstruction of the karyotype of Trachypithecus cristatus (Mammalia: Cercopithecidae). Mol Cytogenet 6:58 (2013).
} 
-Girirajan S, Chen L, Graves T, Marques-Bonet T, Ventura $\mathrm{M}$, et al: Sequencing human-gibbon breakpoints of synteny reveals mosaic new insertions at rearrangement sites. Genome Res 19:178-190 (2009).

- Gribble SM, Ng BL, Prigmore E, Fitzgerald T, Carter NP: Array painting: a protocol for the rapid analysis of aberrant chromosomes using DNA microarrays. Nat Protoc 4:17221736 (2009).

Goodman M: The genomic record of humankind's evolutionary roots. Am J Hum Genet 64:31-39 (1999).

-Gordon L, Yang S, Tran-Gyamfi M, Baggott D, Christensen M, et al: Comparative analysis of chicken chromosome 28 provides new clues to the evolutionary fragility of gene-rich vertebrate regions. Genome Res 17:1603-1613 (2007).

-Jancuskova T, Plachy R, Stika J, Zemankova L, Hardekopf DW, et al: A method to identify new molecular markers for assessing minimal residual disease in acute leukemia patients. Leuk Res 37:1363-1373 (2013).

-Jauch A, Wienberg J, Stanyon R, Arnold N, Tofanelli S, et al: Reconstruction of genomic rearrangements in great apes and gibbons by chromosome painting. Proc Natl Acad Sci USA 89:8611-8615 (1992).

Kehrer-Sawatzki H, Cooper DN: Understanding the recent evolution of the human genome: insights from human-chimpanzee genome comparisons. Hum Mutat 28:99-130 (2007).

-Kehrer-Sawatzki H, Sandig CA, Goidts V, Hameister H: Breakpoint analysis of the pericentric inversion between chimpanzee chromosome 10 and the homologous chromosome 12 in humans. Cytogenet Genome Res 108:9197 (2005).

- Kosyakova N, Hamid AB, Chaveerach A, Pinthong K, Siripiyasing P, et al: Generation of multicolor banding probes for chromosomes of different species. Mol Cytogenet 6:6 (2013).

- Liehr T, Weise A, Hamid AB, Fan X, Klein E, et al: Multicolor FISH methods in current clinical diagnostics. Exp Rev Mol Diagn 13:251255 (2013).

-Locke DP, Archidiacono N, Misceo D, Cardone MF, Deschamps S, et al: Refinement of a chimpanzee pericentric inversion breakpoint to a segmental duplication cluster. Genome Biol 4:R50 (2003).
Misceo D, Capozzi O, Roberto R, Dell'oglio MP, Rocchi M, et al: Tracking the complex flow of chromosome rearrangements from the Hominoidea ancestor to extant Hylobates and $\mathrm{No}$ mascus gibbons by high-resolution synteny mapping. Genome Res 18:1530-1537 (2008).

Mrasek K, Heller A, Rubtsov N, Trifonov V, Starke H, et al: Detailed Hylobates lar karyotype defined by 25 -color FISH and multicolor banding. Int J Mol Med 12:139-146 (2003).

Mrasek K, Schoder C, Teichmann AC, Behr K, Franze B, et al: Global screening and extended nomenclature for 230 aphidicolin-inducible fragile sites, including 61 yet unreported ones. Int J Onc 36:929-940 (2010).

Müller S, Hollatz M, Wienberg J: Chromosomal phylogeny and evolution of gibbons (Hylobatidae). Hum Genet 113:493-501 (2003).

Nickerson E, Nelson DL: Molecular definition of pericentric inversion breakpoints occurring during the evolution of humans and chimpanzees. Genomics 50:368-372 (1998).

O'Brien SJ, Menninger JC, Nash WG: Atlas of Mammalian Chromosomes. John Wiley \& Sons, Danvers (2006).

O'Brien SJ, Yuhki N: Comparative genome organization of the major histocompatibility complex: lessons from the Felidae. Immunol Rev 167:133-144 (1999).

Perelman P, Johnson WE, Roos C, Seuánez HN, Horvath JE, et al: A molecular phylogeny of living primates. PLoS Genet 7:e1001342 (2011).

Peng Q, Pevzner PA, Tesler G: The fragile breakage versus random breakage models of chromosome evolution. PLoS Comput Biol 2:e14 (2006).

- Roberto R, Capozzi O, Wilson RK, Mardis ER, Lomiento M, et al: Molecular refinement of gibbon genome rearrangements. Genome Res 17:249-257 (2007).

Romanenko SA, Perelman PL, Trifonov VA, Graphodatsky AS: Chromosomal evolution in Rodentia. Heredity (Edinburg) 108:4-16 (2012).

Rosen DM, Younkin EM, Miller SD, Casper AM: Fragile site instability in Saccharomyces cerevisiae causes loss of heterozygosity by mitotic crossovers and break-induced replication. PLoS Genet 9:e1003817 (2013).
Stanyon R, Rocchi M, Capozzi O, Roberto R, Misceo D, et al: Primate chromosome evolution: ancestral karyotypes, marker order and neocentromeres. Chromosome Res 16:17-39 (2008).

- Stindl R: The telomeric sync model of speciation: species-wide telomere erosion triggers cycles of transposon-mediated genomic rearrangements, which underlie the saltatory appearance of nonadaptive characters. Naturwissenschaften 101:163-186 (2014).

Tantravahi R, Dev VG, Firschein IL, Miller DA, Miller OJ: Karyotype of the gibbons Hylobates lar and H. moloch inversion in chromosome 7. Cytogenet Cell Genet 15:92-102 (1975).

- Trifonov VA, Musilova P, Kulemsina AI: Chromosome evolution in Perissodactyla. Cytogenet Genome Res 137:208-217 (2012).

Villa O, Mallo M, Kosyakova N, Salido M, Liehr $\mathrm{T}$, et al: Deletion of TET2 gene in an acute myeloid leukemia case with at $(4 ; 15)(\mathrm{q} 24 ; \mathrm{q} 26)$ characterized by glass needle based chromosome microdissection and oligonucleotide array. Leuk Res 35:e161-163 (2011).

von Grotthuss M, Ashburner M, Ranz JM: Fragile regions and not functional constraints predominate in shaping gene organization in the genus Drosophila. Genome Res 20:1084-1096 (2010).

Weise A, Starke H, Mrasek K, Claussen U, Liehr T: New insights into the evolution of chromosome 1. Cytogenet Genome Res 108:217-222 (2005)

Weise A, Mrasek K, Fickelscher I, Claussen U, Cheung SW, et al: Molecular definition of high-resolution multicolor banding probes: first within the human DNA sequence anchored FISH banding probe set. J Histochem Cytochem 56:487-493 (2008).

-Wilson TE, Arlt MF, Park SH, Rajendran S, Paulsen $\mathrm{M}$, et al: Large transcription units unify copy number variants and common fragile sites arising under replication stress. Genome Res. 25:189-200 (2015).

-Yang F, Trifonov V, Ng BL, Kosyakova N, Carter NP: Generation of paint probes by flow-sorted and microdissected chromosomes, In Liehr T (ed): Fluorescence In Situ Hybridization (FISH) - Application Guide, pp 35-52 (Springer, Berlin 2009).

Zhao S, Shetty J, Hou L, Delcher A, Zhu B, et al: Human, mouse, and rat genome large-scale rearrangements: stability versus speciation. Genome Res 14:1851-1860 (2004).
Comprehensive Karyotype of

White-Handed Gibbon
Cytogenet Genome Res 2015;145:42-49 DOI: $10.1159 / 000381764$ 